\title{
Case Report \\ Sertoli-Leydig Cell Tumor with Concurrent Rhabdomyosarcoma: Three Case Reports and a Review of the Literature
}

\author{
Trisha Plastini and Arthur Staddon \\ Pennsylvania Hospital, Penn Medicine, Philadelphia, PA, USA \\ Correspondence should be addressed to Trisha Plastini; trisha.sterlicchi@uphs.upenn.edu \\ Received 31 January 2017; Revised 19 May 2017; Accepted 29 May 2017; Published 2 July 2017 \\ Academic Editor: Gottfried J. Locker
}

Copyright (C) 2017 Trisha Plastini and Arthur Staddon. This is an open access article distributed under the Creative Commons Attribution License, which permits unrestricted use, distribution, and reproduction in any medium, provided the original work is properly cited.

\begin{abstract}
Sertoli-Leydig Cell Tumors (SLCTs) make up $<1 \%$ of all ovarian tumors and are benign or malignant, androgen-secreting tumors. Rhabdomyosarcoma (RMS) is a heterogeneous group of malignant tumors that resemble developing skeletal muscle. There have been case reports of patients with concurrent SLCT and RMS with limited treatment options. We aim to demonstrate treatment strategies used in our patients, which seemed to have prolonged survival when compared to prior case reports of patients not cured by surgical resection. Herein we describe 22 cases of SLCT with RMS elements as discussed in prior case reports and three cases from the authors' institution. Of the 19 cases from prior case reports, five were lost to follow-up and two had NED after surgical intervention. Eleven patients had recurrence and were deceased within one year. Of those patients not surgically cured, only three patients were documented as living beyond two years, all of whom received chemotherapy. The three patients presented from our institution had clinical evidence of response to chemotherapy that is traditionally used for RMS. In conclusion, chemotherapy with doxorubicin and ifosfamide has activity in patients with SLCT and RMS as does salvage chemotherapy with vincristine, irinotecan, and temozolomide.
\end{abstract}

Sertoli-Leydig Cell Tumors (SLCTs), also known as arrhenoblastomas, make up $<0.5-1 \%$ of all ovarian tumors and are benign or malignant, androgen-secreting tumors originating from the ovarian stromal sex cords [1]. In up to half of cases, SLCT can be associated with signs of virilization, such as hirsutism and amenorrhea, and SLCTs occur more often in women of reproductive age $[1,2]$. There are five histological subtypes of SLCT as follows: well differentiated, intermediate differentiation, poorly differentiated, retiform, and heterologous or mixed [3-5]. Twenty percent of SLCTs are variants with heterologous elements, which are mostly benign gastrointestinal epithelium, but 5\% of SLCTs contain heterologous mesenchymal elements [4]. Ultrasound is the primary imaging modality to identify adnexal masses. Serum levels of AFP and $\beta$-hCG may be elevated in patients with SLTC [2]. SLTC immunocytochemical characterization is positive for testosterone and estradiol of the Sertoli and Leydig cells [1]. A mutation of the DICER1 gene has been associated with SLCTs in up to $63 \%$ of patients, and patients with this mutation should be screened for thyroid disease [6, 7]. The immunohistochemical testing of antibodies against alpha-inhibin and myogenin is needed to diagnose rhabdomyosarcoma (RMS) and accurately grade the SLCT [8]. The TMN and FIGO stages are listed in Table 1. The treatment and prognosis of SLCT depend on the patient's age, tumor stage, and degree of tumor differentiation $[1,2]$. Various types of surgery with or without adjuvant chemotherapy are the primary treatment modalities for treatment of SLCT [2]. Malignant SLCT ten-year survival rates are approximately $87 \%$ for intermediate differentiation and $41 \%$ for poor differentiation [9].

Rhabdomyosarcoma (RMS) is a heterogeneous group of malignant tumors that resemble developing skeletal muscle [10-12]. These tumors are 1.4 times more common in males without differences among races or ethnic groups [13]. RMS is the most common soft tissue sarcoma of childhood, and common sites of primary disease include the head and neck area, genitourinary tract, and extremities $[10,11]$. 


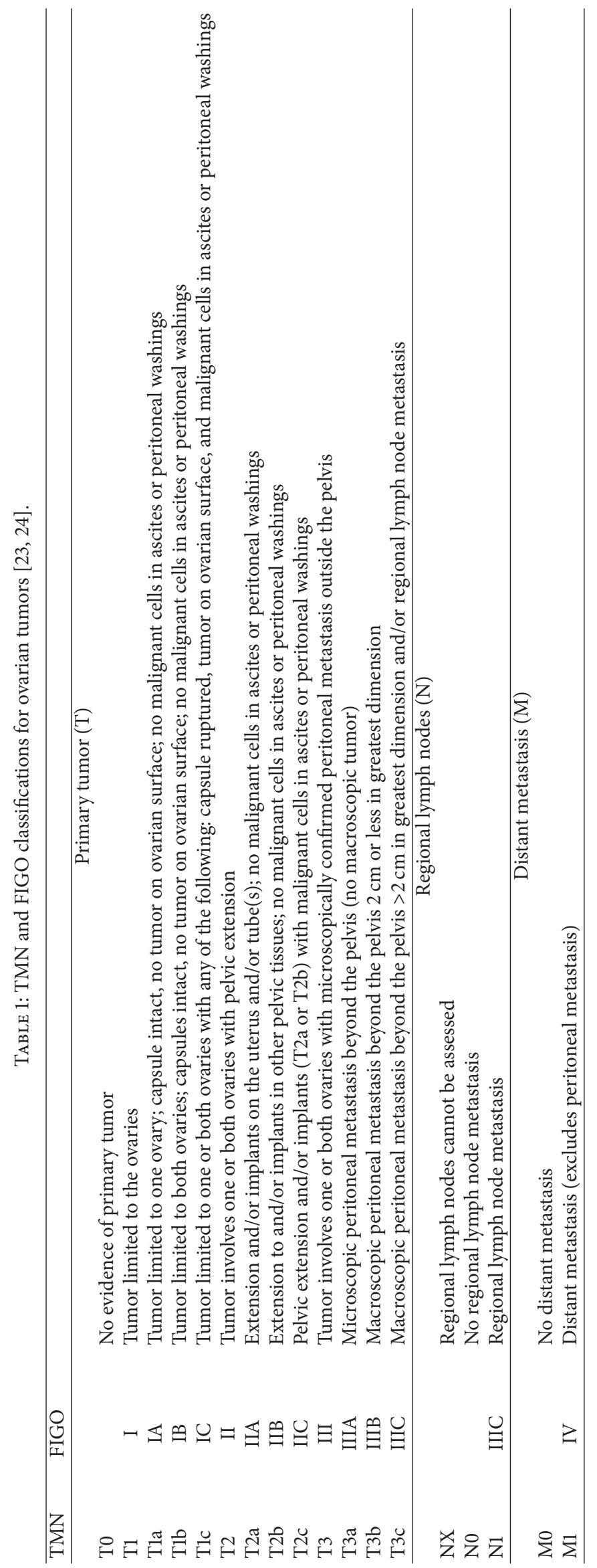


Most cases of RMS appear to be sporadic, but the disease has been associated with familial syndromes such as LiFraumeni syndrome and neurofibromatosis [10]. There are two histologic subtypes of RMS, embryonal and alveolar; Alveolar RMS is more aggressive with small, round cells while embryonal RMS has a more favorable prognosis with spindle-shaped cells with a stromal-rich appearance [10, 11, 14]. Embryonal variants include leiomyomatous RMS that is predominantly of paratesticular origin and the botryoid variant with subepithelial aggregates of tumor cells known as the cambium layer [14]. Sarcoma botryoides can arise from the mucosal surfaces of the cervix, bladder, or vagina [15]. Radiologic evaluation of RMS should include CT scan or MRI of the primary and surrounding structures. Treatment approaches to RMS incorporate chemotherapy, radiation therapy, and surgery based on risk stratification. Complete surgical resection is considered if it will not be cosmetically damaging. The five-year overall survival rate for adults with RMS is approximately $27 \%$ [16].

The association between cervical sarcoma botryoides and ovarian SCLT has been described in at least eight patients from prior case reports [15, 17-22]. Adjuvant ifosfamide and epiadriamycin were used in one of these patients who had recurrence of uterine disease after 6 years, and conservative polypectomy was encouraged by some authors [20]. A genetic linkage to abnormalities on chromosome 12 was suggested by one paper [19]. One patient with concurrently diagnosed pleuropulmonary blastoma and embryonal rhabdomyosarcoma of the cervix had a DICER1 germline mutation [21]. This patient was treated with vincristine, actinomycin, and cyclophosphamide with recurrence of SLCT 7 years later [21].

Herein, we report three cases of patients with ovarian SLCT with RMS components. To our knowledge, there have been cases reported in literature with limited treatment options. We aim to demonstrate treatment strategies used in our patients, which seemed to have prolonged their lives when compared to prior case reports of patients not cured by surgical resection, in order to provide treatment options for clinicians treating patients with these concurrent rare malignancies.

Patient 1 was a 32-year-old Caucasian female noted to have a left sided ovarian mass on CT scan after presenting for left lower quadrant abdominal pain. She was status post resection and pathology was consistent with stage Ia poorly differentiated SLCT. Five months later, recurrent pains lead to a trans-vaginal US that showed a right pelvic mass. She underwent radical hysterectomy and debulking procedure that showed pathology consistent with recurrent poorly differentiated SLCT with heterologous RMS. A follow-up PET scan showed uptake bilaterally in the pelvis. A month later, she was started on doxorubicin, ifosfamide, and vincristine. She was hospitalized for a small bowel obstruction with stable metastatic abdominal nodules on CT. She received cycle 2 doxorubicin, ifosfamide, and vincristine while hospitalized and had a palliative PEG placed. Her chemotherapy was changed to ifosfamide and etoposide because of mucositis and palmar-plantar erythrodysesthesia. After a total of 6 cycles of chemotherapy, she was found to be in complete clinical remission by CT. Approximately 10 months later, CT showed a liver lesion and pelvic mass consistent with recurrent poorly differentiated SLCT on repeat biopsy with no RMS elements seen. She was given 3 cycles of bleomycin, etoposide, and cisplatin (BEP). Bleomycin was held for cycles 4 through 8 due to side effects. A follow-up PET showed stable disease. Three months later, she was found to have a left lower quadrant mass consistent with recurrent SLCT at which point radiation therapy was given with good response with only a small amount of residual disease. Eight months later, a biopsy of a liver lesion was consistent with recurrent SLCT with no definitive RMS identified. She underwent further radiation with stable disease for approximately five months before repeat imaging showed marked disease progression with symptoms that lead to hospice care. The time from diagnosis until death was approximately four years.

Patient 2 was a 30 -year-old, black female with a left sided ovarian mass found incidentally during a cesarean section that was initially called a granulosa tumor on pathology but later deemed SLCT with focal poor differentiation when reviewed. At the time of initial diagnosis she was thought to have stage IA disease that was cured surgically. She was lost to follow-up for 13 months at which time a follow-up CT showed a right sided ovarian mass that increased on serial imaging. Surgery for recurrence revealed a left adnexal mass, left pelvic sidewall, cul-de-sac, and anterior abdominal wall nodules with pathology consistent with high-grade sarcoma ultimately deemed embryonal RMS consistent with recurrence of the poorly differentiated SLCT. Two months later, she was started on doxorubicin, ifosfamide, and vincristine for a total of 6 cycles with follow-up CT showing NED. A repeat CT performed 7 months after chemotherapy completion showed an increasing pelvic mass that was resected. Pathology was consistent with recurrent SLCT with RMS, and a liver mass was discovered during surgery that was not seen on CT scan. She was started on salvage chemotherapy for RMS with vincristine, irinotecan, and temozolomide for a total of 6 cycles. Follow-up CT after cycle 4 showed NED. The patient developed massive progression 5 months after her last cycle of chemotherapy with small bowel obstruction requiring debulking surgery with colostomy placement and ultimately hospice care. The time span from diagnosis until death was approximately 3.5 years.

Patient 3 was a 23-year-old female who presented with nausea and vomiting with an US showing a right ovarian mass. She underwent exploratory laparotomy with debulking and removal of a large abdominal pelvic mass with a right salpingo-oophorectomy (RSO) and appendectomy. Pathology was consistent with stage IA poorly differentiated SLCT with some elements of RMS, and she was found to have a DICER1 mutation. She underwent 6 cycles of doxorubicin, ifosfamide, and vincristine and is currently NED on followup imaging approximately 10 months after chemotherapy was initiated.

In one case report, a patient with SLCT of intermediate differentiation and RMS of FIGO stage $1 \mathrm{C}$ was treated with surgery alone and remained with no evidence of disease (NED) at four years after surgery [8]. These authors report 


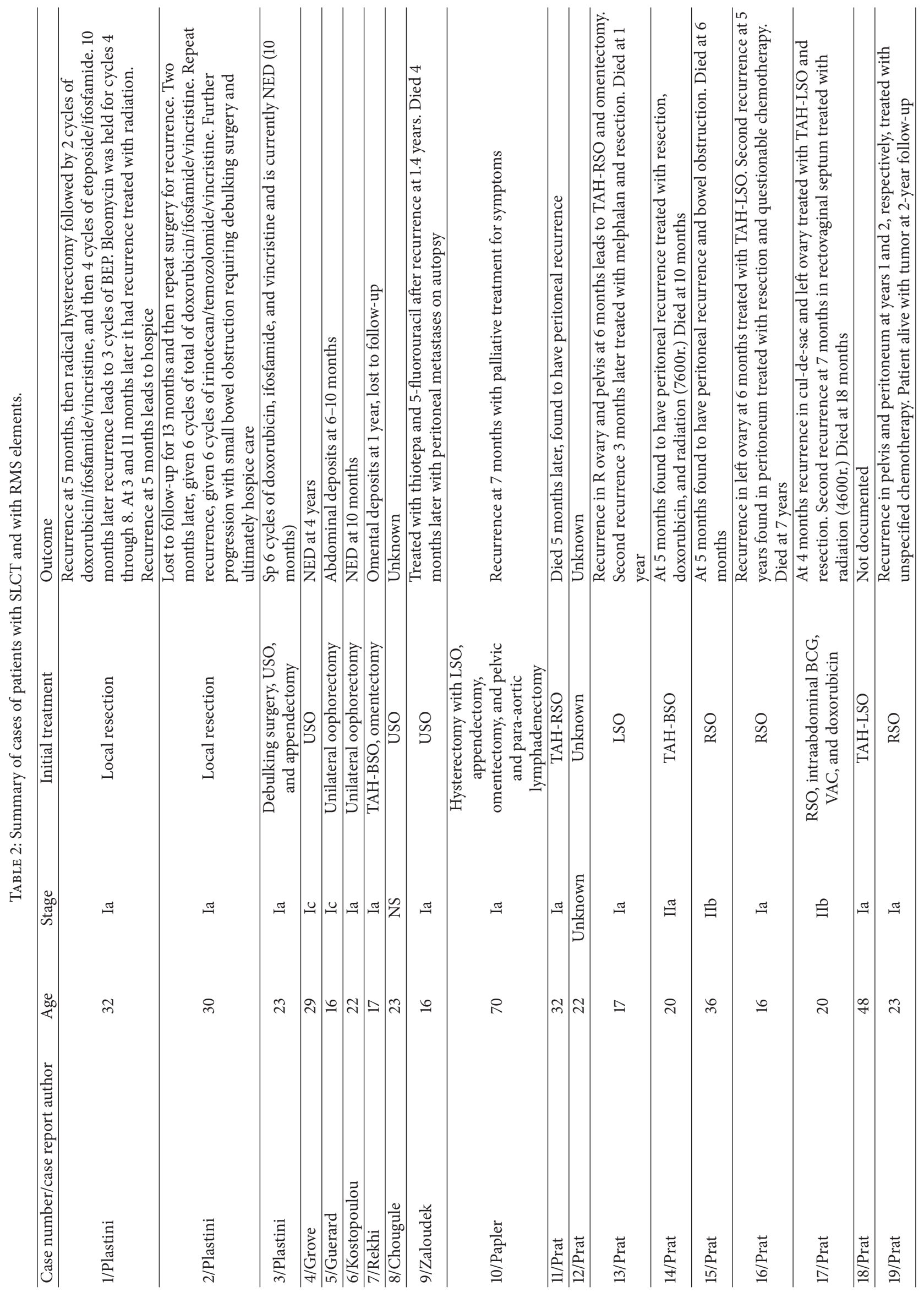


two similar case reports with SLCT of intermediate differentiation and RMS where one patient was found to have NED at ten months after unilateral salpingo-oophorectomy (USO) for stage IA disease while another patient with capsular invasion was initially treated with oophorectomy alone but had reoccurrences at months six and ten, at which time she was treated with unspecified adjuvant chemotherapy $[8,25$, 26].

Another case report describes a patient who after a unilateral oophorectomy was found to have an SLCT with focal rhabdomyoblastic differentiation, and she developed an omental mass with features consistent with high-grade sarcoma one year later. She underwent further surgery with a plan for adjuvant chemotherapy but was lost to follow-up [27]. Chougule et al. report a case where ovarian SLCT with RMS and borderline mucinous neoplasm was found in a 26year-old patient after USO but no further information on treatment or follow-up was provided [28].

Zaloudek and Norris described a 16-year-old patient with stage Ia poorly differentiated SLCT with RMS elements who was treated with USO and later thiotepa and 5-fluorouracil after recurrence at 1.4 years. She was found to have peritoneal metastases on autopsy after her death four months later [29].

The most recent case report of SLCT with RMS elements describes a 70-year-old patient who was diagnosed with stage Ia disease and underwent hysterectomy with left salpingo-oophorectomy (LSO), appendectomy, and omentectomy without evidence of macroscopic disease after the procedures. At 7 months postoperatively, she represented with a large abdominal mass, ascites, unilateral hydronephrosis, and a massive pulmonary embolism. She was treated palliatively and died 15 days later without subsequent autopsy performance [30].

The largest number of case reports was presented by Prat et al. who document nine cases of SLCT with skeletal muscle elements. With one exception, all of these subjects were initially treated with surgery alone. Of those with available information, all subjects had recurrence within one year of initial treatment. Most subjects had recurrence surgically treated and most were deceased within one year. For full information on these subjects, see Table 2 [31].

Discussion. Herein we describe 19 cases of SLCT with RMS elements as discussed in prior case reports as well as three cases from the authors' institution. Of these 19 cases, five were lost to follow-up and two were documented as being NED after surgical intervention alone. Of the remaining cases, eleven patients had recurrence within one year and five of these patients were deceased within that year. Of those patients not surgically cured, only three patients were documented as living beyond two years, and all three of these patients received chemotherapy.

In patients with RMS, chemotherapy regimens considered in recurrent disease include vincristine, doxorubicin, and cyclophosphamide; ifosfamide and etoposide; and vincristine, irinotecan, and temozolomide [32, 33]. Standard adjuvant chemotherapy regimens include cisplatin based combinations with etoposide/bleomycin or etoposide/ifosfamide [6]. The three patients presented from our institution with concurrent SLCT and RMS all had clinical evidence of response to chemotherapy that is traditionally used for RMS on follow-up imaging. In conclusion, chemotherapy with doxorubicin and ifosfamide has activity in patients with concurrent SLCT and RMS as does salvage chemotherapy with vincristine, irinotecan, and temozolomide.

\section{Conflicts of Interest}

The authors declare that they have no conflicts of interest.

\section{References}

[1] A. Gheorghisan-Galateanu, S. Fica, D. C. Terzea, A. Caragheorgheopol, and V. Horhoianu, "Sertoli-Leydig cell tumor-A rare androgen secreting ovarian tumor in postmenopausal women: case report and review of literature," Journal of Cellular and Molecular Medicine, vol. 7, no. 4, pp. 461-471, 2003.

[2] M. Horta, T. M. Cunha, R. C. Marques, and A. Félix, "Ovarian Sertoli-Leydig cell tumor with heterologous elements of gastrointestinal type associated with elevated serum alphafetoprotein level: an unusual case and literature review," Journal of Radiology Case Reports, vol. 8, no. 11, pp. 30-41, 2014.

[3] R. H. Young, "Sertoli-Leydig cell tumors of the ovary: review with emphasis on historical aspects and unusual variants," International Journal of Gynecological Pathology, vol. 12, no. 2, pp. 141-147, 1993.

[4] R. H. Young and R. E. Scully, "Ovarian Sertoli-Leydig cell tumors. A clinicopathological analysis of 207 cases," American Journal of Surgical Pathology, vol. 9, no. 8, pp. 543-569, 1985.

[5] R. H. Young, J. Prat, and R. E. Scully, "Ovarian Sertoli-Leydig cell tumors with heterologous elements I. Gastrointestinal epithelium and carcinoid: a clinicopathologic analysis of thirty-six cases," Cancer, vol. 50, no. 11, pp. 2448-2456, 1982.

[6] D. T. Schneider, D. Orbach, G. Cecchetto et al., "Ovarian Sertoli Leydig cell tumours in children and adolescents: an analysis of the European Cooperative Study Group on pediatric rare tumors (EXPeRT)," European Journal of Cancer, vol. 51, no. 4, pp. 543-550, 2015.

[7] N. Conlon, A. M. Schultheis, S. Piscuoglio et al., "A survey of DICER1 hotspot mutations in ovarian and testicular sex cordstromal tumors," Modern Pathology, vol. 28, no. 12, pp. 16031612, 2015.

[8] A. Grove and V. Vestergaard, "Ovarian Sertoli-Leydig cell tumor of intermediate grade with heterologous elements of rhabdomyosarcoma. A case report and a review of the literature," Annals of Diagnostic Pathology, vol. 10, no. 5, pp. 288-293, 2006.

[9] W. P. McGuire, W. J. Hoskins, M. F. Brady et al., "Cyclophosphamide and cisplatin compared with paclitaxel and cisplatin in patients with stage III and stage IV ovarian cancer," International Journal of Gynecology \& Obstetrics, vol. 334, 1, no. 1, pp. 6-86, 1996.

[10] R. Dagher and L. Helman, "Rhabdomyosarcoma: an overview," Oncologist, vol. 4, no. 1, pp. 34-44, 1999.

[11] C. Wang, "Childhood rhabdomyosarcoma: recent advances and prospective views," Journal of Dental Research, vol. 91, no. 4, pp. 341-350, 2012.

[12] D. Egas-Bejar and W. W. Huh, "Rhabdomyosarcoma in adolescent and young adult patients: current perspectives," Adolescent Health, Medicine and Therapeutics, vol. 5, pp. 115-125, 2014. 
[13] J. C. Breneman, E. Lyden, A. S. Pappo et al., "Prognostic factors and clinical outcomes in children and adolescents with metastatic rhabdomyosarcoma-a report from the intergroup rhabdomyosarcoma study IV," Journal of Clinical Oncology, vol. 21, no. 1, pp. 78-84, 2003.

[14] W. A. Newton, E. A. Gehan, B. L. Webber et al., "Classification of rhabdomyosarcomas and related sarcomas. Pathologic aspects and proposal for a new classification-an intergroup rhabdomyosarcoma study," Cancer, vol. 76, no. 6, pp. 1073-1085, 1995.

[15] L. Pommert and W. Bradley, "Pediatric gyecologic cancers," Current Oncology Reports, vol. 19, article 44, 2017.

[16] I. Sultan, I. Qaddoumi, S. Yaser, C. Rodriguez-Galindo, and A. Ferrari, "Comparing adult and pediatric rhabdomyosarcoma in the surveillance, epidemiology and end results program, 1973 to 2005: an analysis of 2,600 patients," Journal of Clinical Oncology, vol. 27, no. 20, pp. 3391-3397, 2009.

[17] D. A. Daya and R. E. Scully, "Sarcoma botryoides of the uterine cervix in young women: a clinicopathological study of 13 cases," Gynecologic Oncology, vol. 29, no. 3, pp. 290-304, 1988.

[18] P. Golbang, A. Khan, J. Scurry, I. MacIsaac, and R. Planner, "Cervical sarcoma botryoides and ovarian Sertoli-Leydig cell tumor," Gynecologic Oncology, vol. 67, no. 1, pp. 102-106, 1997.

[19] G. E. McClean, S. Kurian, N. Walter, A. Kekre, and W. G. McCluggage, "Cervical embryonal rhabdomyosarcoma and ovarian Sertoli-Leydig cell tumour: a more than coincidental association of two rare neoplasms?" Journal of Clinical Pathology, vol. 60, no. 3, pp. 326-328, 2007.

[20] P. Rosenberg, S. Carinelli, M. Peiretti, V. Zanagnolo, and A. Maggioni, "Cervical sarcoma botryoides and ovarian SertoliLeydig cell tumor: a case report and review of literature," Archives of Gynecology and Obstetrics, vol. 285, no. 3, pp. 845848, 2012.

[21] L. P. Dehner, J. A. Jarzembowski, and D. A. Hill, "Embryonal rhabdomyosarcoma of the uterine cervix: a report of 14 cases and a discussion of its unusual clinicopathological associations," Modern Pathology, vol. 25, no. 4, pp. 602-614, 2012.

[22] J. P. Panagiotou, S. Polychronopoulou, K. Sofou, C. VanVlietConstantinidou, E. Papandreou, and S. Haidas, "Second and third malignant solid tumor in a girl with ovarian Sertoli-Leydig tumor," Pediatric Blood and Cancer, vol. 46, no. 5, pp. 654-656, 2006.

[23] J. Prat and FIGO Committee on Gynecologic Oncology, "FIGO Guidelines. Staging classification for cancer of the ovary, fallopian tube, and peritoneum," International Journal of Gynecology \& Obstetrics, vol. 124, no. 1, pp. 1-5, 2014.

[24] NCCN (National Comprehensive Cancer Network), "NCCN clinical practice guidelines in oncology: (NCCN guidelines $\left.{ }^{\circledR}\right)$ ovarian cancer including fallopian tube cancer and primary peritoneal cancer. Version 1," 2015, http://www.nccn.org/professionals/physician_gls/pdf/ovarian.pdf.

[25] M. J. Guérard, A. Ferenczy, and M. A. Arguelles, "Ovarian Sertoli-Leydig cell tumor with rhabdomyosarcoma: an ultrastructural study," Ultrastructural Pathology, vol. 3, no. 4, pp. 347-358, 1982.

[26] E. Kostopoulou and A. Talerman, "Ovarian Sertoli-Leydig cell tumor of intermediate differentiation with immature skeletal muscle heterologous elements," Acta Obstetricia et Gynecologica Scandinavica, vol. 82, no. 2, pp. 197-198, 2003.

[27] B. Rekhi, A. Karpate, K. K. Deodhar, and R. F. Chinoy, "Metastatic rhabdomyosarcomatous elements, mimicking a primary sarcoma, in the omentum, from a poorly differentiated ovarian Sertoli-Leydig cell tumor in a young girl: an unusual presentation with a literature review," Indian Journal of Pathology and Microbiology, vol. 52, no. 4, pp. 554-558, 2009.

[28] A. Chougule, P. Singh, P. K. Saha, and P. Dey, "Ovarian SertoliLeydig cell tumour with rhabdomyosarcoma and borderline mucinous neoplasm," Pathology, vol. 48, no. 3, pp. 278-281, 2016.

[29] C. Zaloudek and H. J. Norris, "Sertoli-Leydig tumors of the ovary. A clinicopathologic study of 64 intermediate and poorly differentiated neoplasms," American Journal of Surgical Pathology, vol. 8, no. 6, pp. 405-418, 1984.

[30] T. B. Papler, S. F. Grazio, and B. Kobal, "Sertoli-Leydig cell tumor with retiform areas and overgrowth of rhabdomyosarcomatous elements: case report and literature review," Journal of Ovarian Research, vol. 9, no. 1, article 46, 2016.

[31] J. Prat, R. H. Young, and R. E. Scully, "Ovarian Sertoli-Leydig cell tumors with heterologous elements. II. Cartilage and skeletal muscle: a clinicopathologic analysis of twelve cases," Cancer, vol. 50, no. 11, pp. 2465-2475, 1982.

[32] B. A. Mixon, M. J. Eckrich, S. Lowas, and M. E. Engel, "Vincristine, irinotecan, and temozolomide for treatment of relapsed alveolar rhabdomyosarcoma," Journal of Pediatric Hematology/Oncology, vol. 35, no. 4, pp. e163-e166, 2013.

[33] S. Winter, S. Fasola, H. Brisse, V. Mosseri, and D. Orbach, "Relapse after localized rhabdomyosarcoma: evaluation of the efficacy of second-line chemotherapy," Pediatric Blood and Cancer, vol. 62, no. 11, pp. 1935-1941, 2015. 


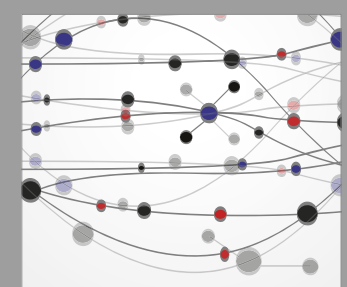

The Scientific World Journal
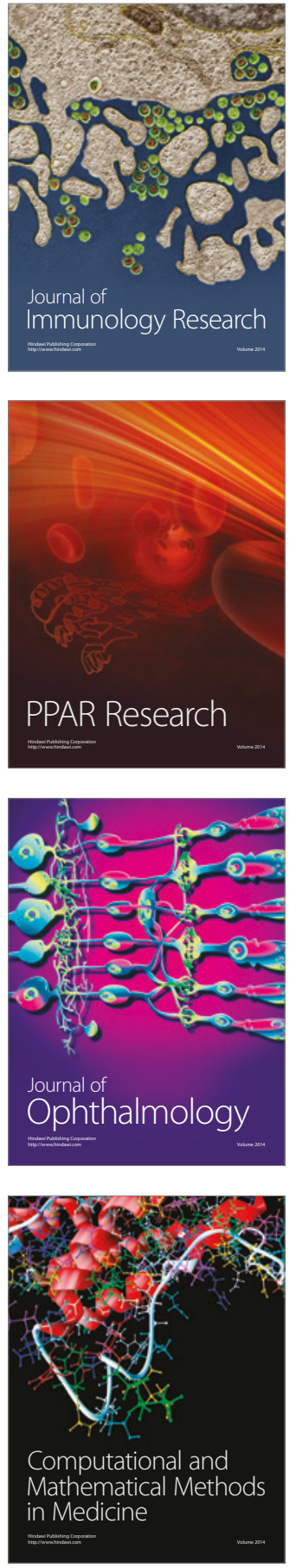

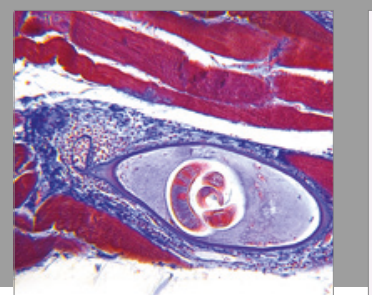

Gastroenterology Research and Practice
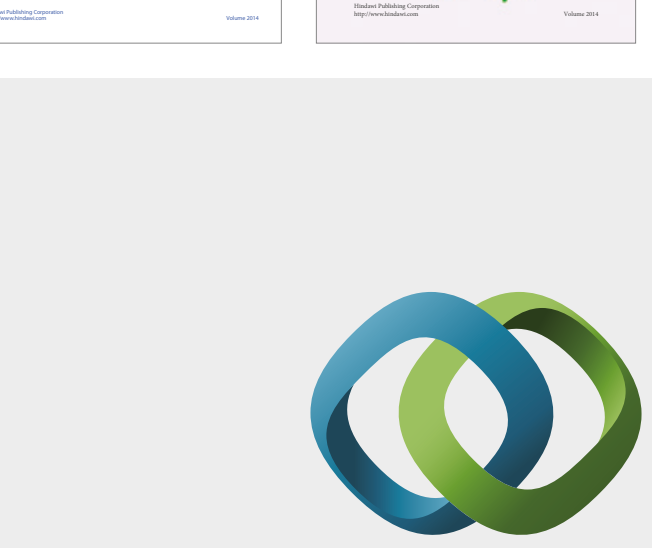

\section{Hindawi}

Submit your manuscripts at

https://www.hindawi.com
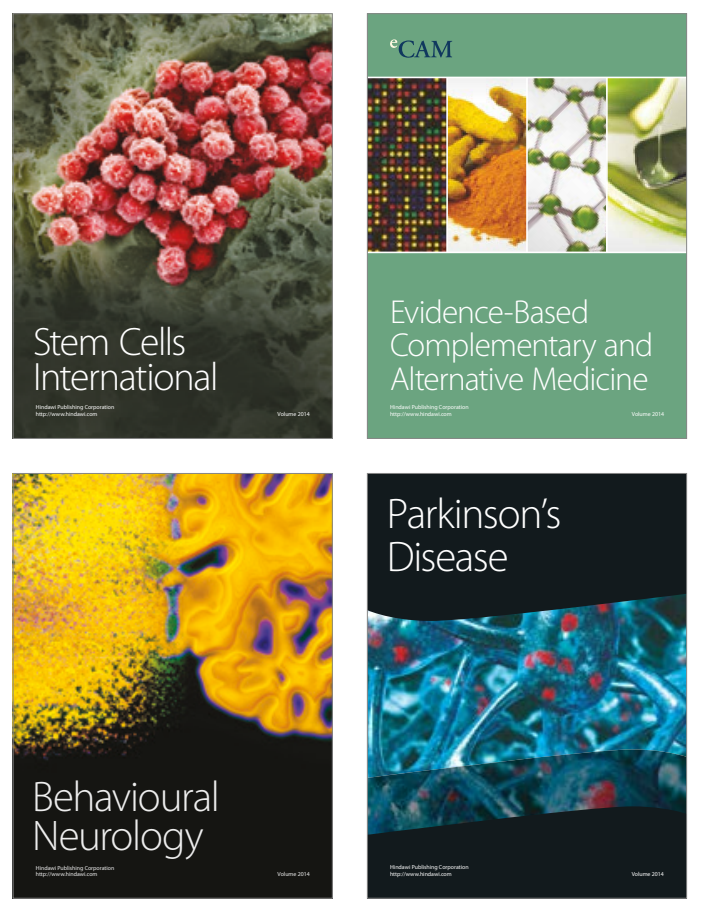
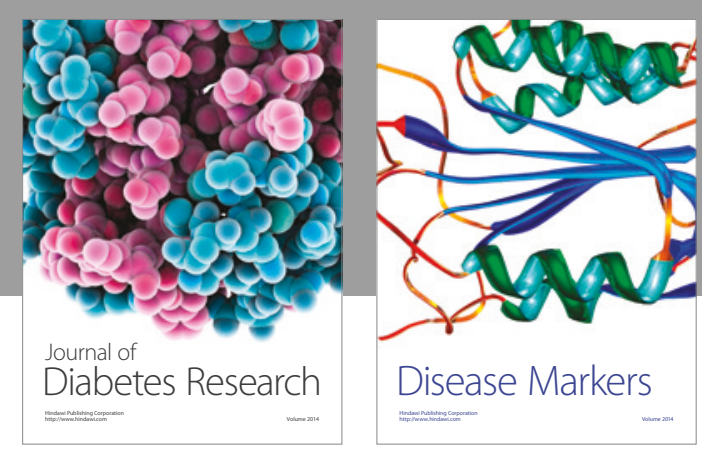

Disease Markers
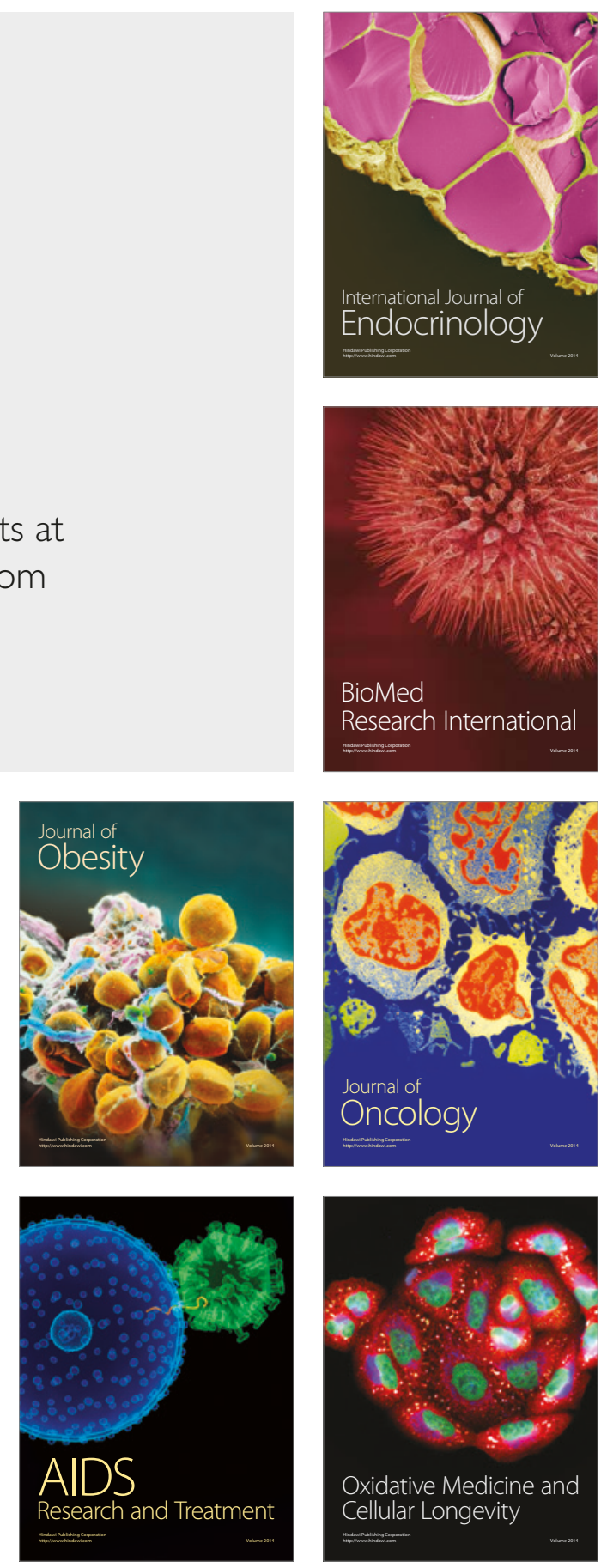